\title{
Cluster formation versus star formation rates around six regions in the Large Magellanic Cloud
}

\begin{abstract}
A. Subramaniam
Indian Institute of Astrophysics, II Block, Koramangala, Bangalore 560034, India

e-mail: purni@iiap.ernet.in

Received 5 January 2004 / Accepted 15 June 2004

Abstract. The stellar population and star clusters around six regions in the Large Magellanic Cloud (LMC) are studied to understand the correlation between star formation and cluster formation rates. We used the stellar data base of the OGLE II LMC survey and the star cluster catalogues. The observed distributions of stellar density in the colour-magnitude diagrams (CMDs) were compared with synthetic ones generated from stellar evolutionary models. By minimising the reduced $\chi^{2}$ values, the star formation history of the regions were obtained in terms of star formation rates (SFR). All the regions were found to show large SFRs between the ages 500-2 Gyr with lower values for younger and older ages. A correlated peak in the cluster and SFRs is found for ages $\sim 1 \mathrm{Gyr}$, and for ages less than 100 Myr. Five of the six regions show significant cluster formation in the age range of 100-300 Myr, when the SFRs were found to be very low. This indicates anti-correlation between star and cluster formation rates for the 100-300 Myr age range. A possible reason may be that the stars are predominantly formed in clusters, whether bound or unbound, as a result of star formation during the above age range. The enhanced cluster formation rate in the 100-300 Myr age range could be correlated with the encounter of the LMC with the Small Magellanic Cloud, while the enhanced star and cluster formation at $\sim 1 \mathrm{Gyr}$ does not correspond to any interaction. This could indicate that the star formation induced by interactions is biased towards group or cluster formation of stars.
\end{abstract}

Key words. galaxies: Magellanic Clouds - galaxies: star clusters - stars: formation

\section{Introduction}

In recent years, the Large Magellanic Cloud (LMC) has been very thoroughly studied using various surveys, for example, OGLE II (Udalski et al. 2000), Magellanic Clouds Photometric Survey (Zaritsky et al. 1997). These surveys were used partly or fully to study the star clusters and the stellar population in the LMC. However, the spatial correlation between the star and cluster formation on small scales have not been studied. Girardi et al. (1995) used the star cluster catalogue of Bica et al. (1996) to derive the star cluster properties in the LMC. Pietrzynski \& Udalski (2000) used the OGLE II data and studied the age distribution of the LMC star clusters. There were also studies of star clusters and stellar population around them, (for example, Olsen et al. 1998; Olsen 1999), but these also do not compare the spatial correlation between cluster and star formation episodes. The recent study by Holtzman et al. (1999) suggested that the star formation history of the field stars is different from that of the clusters. This difference is seen in the age range 4.0-12 Gyr, where there seems to be a paucity in cluster formation. It is concluded that in general, the star clusters in the LMC are not good tracers of the stellar population (van den Bergh 1999). This conclusion has been made from the analysis of the cluster and stellar population in the whole of the LMC. In this study, an attempt is made to study the correlation between the star formation and cluster formation episodes around a few regions in the LMC. Also, the emphasis is on the younger age range, for ages $\leq 1 \mathrm{Gyr}$ and at smaller scales.

The recent star forming regions, like 30 Dor and the super giant shells found in the LMC indicate that the star formation which began at one point propagates to larger distances in the LMC. The theories put forward to explain these structures include stochastic self-propagating star formation, SSPSF (Feitzinger et al. 1981) and bow-shock induced star formation (de Boer et al. 1998). A correlation between the events of cluster formation and star formation is expected in the resulting stellar population. We explore this correlation in this study, by looking at star clusters surrounding a few regions in the LMC.

\section{Data}

\subsection{Regions}

The regions studied here were chosen for a different project, that is, to study the stellar population around novae in the LMC (see Subramaniam \& Anupama 2002). From the above study, six regions were selected for the present analysis. The stellar data within a radius of a few arcmin are used to study the star formation history (SFH) of the region under consideration, whereas star clusters are identified within 30 arcmin 
Table 1. Location of the six regions in the Large Magellanic Cloud.

\begin{tabular}{lllllll}
\hline \hline Region & \multicolumn{3}{c}{ RA } & \multicolumn{3}{l}{ Dec } \\
& $\mathrm{h}$ & $\mathrm{m}$ & $\mathrm{s}$ & $\circ$ & \multicolumn{1}{l}{${ }^{\prime}$} \\
\hline Region 1 & 05 & 35 & 29.33 & -70 & 21 & 29.39 \\
Region 2 & 05 & 32 & 09.27 & -70 & 22 & 11.70 \\
Region 3 & 05 & 26 & 50.33 & -70 & 01 & 23.08 \\
Region 4 & 05 & 23 & 50.12 & -70 & 00 & 23.50 \\
Region 5 & 05 & 23 & 21.82 & -69 & 29 & 48.48 \\
Region 6 & 05 & 08 & 01.10 & -68 & 37 & 37.67 \\
\hline
\end{tabular}

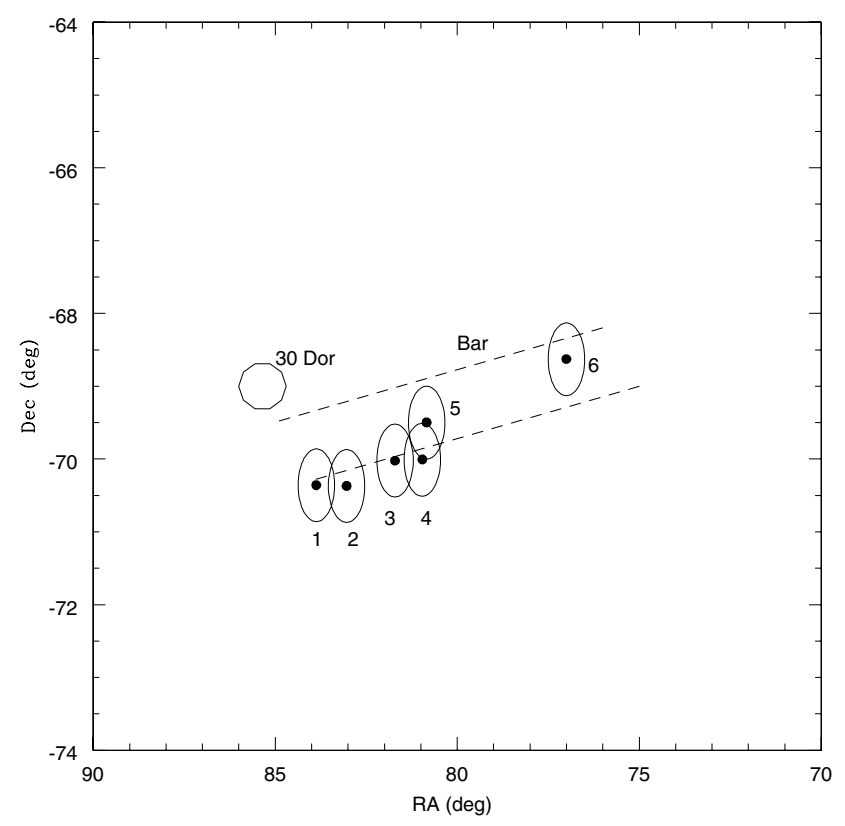

Fig. 1. The locations of the regions studied. The Bar and 30 Dor are also shown.

radius to study the cluster formation events. The above value of 30 arcmin is chosen such that the area covered is similar to the size of supergiant shells in the LMC. The regions selected here satisfy the following two conditions - a) there is good number of star clusters within 30 arcmin radius and b) ages are known for most of the identified clusters. The locations of these regions are given in Table 1 . These locations are also plotted in Fig. 1. The dots show the centers of the regions studied and the big circles around them show the extent of the region scanned for star clusters.

Field stars within a radius of a few arcmin around these regions were identified from the OGLE II survey (Udalski et al. 2000). We used the photometric data in the $V$ and $B$ pass bands, and $V$ vs. $(B-V)$ colour-magnitude diagrams (CMDs) of the identified field stars were used for further analyses.

\subsection{Star clusters}

The star clusters in the vicinity of six regions were identified and their properties obtained based on the following catalogues: Pietrzynski et al. (1999) (P99), Bica et al. (1999) (B99), Bica et al. (1996) (B96), Pietrzynski \& Udalski (2000) (PU2000). B96 presented integrated $U B V$ photometry of 624 star clusters and associations in the LMC. They estimated the ages of the clusters based on their integrated colours and hence classified the clusters into SWB types (Searle et al. 1980), which is basically an age sequence. This classification can be used to obtain the approximate age of the clusters. B99 is a revised version of the above catalogue and contains about 1808 star clusters for which the positions and extents are tabulated. P99 presented photometric data of 745 star clusters and their nearby field, of which 126 are new. PU2000 estimated the ages for 600 star clusters presented in the P99 catalogue. The catalogues in B99 and P99 were used to identify the clusters, while B96 and PU2000 were used to estimate the ages of the identified star clusters.

Clusters have been identified within an area of $30 \mathrm{arcmin}$ radius around 6 regions. 109 clusters have been identified near these regions. Of these, age estimates for 89 clusters could be obtained from PU2000 and B96. B96 gives the age of the cluster in terms of groups. Since the interest is in age groups of the cluster population rather than the ages of the individual clusters, the above data serve the purpose. Therefore, even those clusters whose exact age is known are also grouped. No systematic shift in the cluster age is found between the two catalogues. The number of clusters detected near each region, the number for which the age is known and the number of clusters in various age groups are tabulated in Table 2.

Field stars within a radius of a few arcmin are analysed to study the star formation history, while clusters within $30 \operatorname{arcmin}(\sim 400 \mathrm{pc})$ radius are considered to identify the cluster formation episodes. The choice of a larger radius for the clusters is justified as they are being used to study the star formation events which took place on relatively larger scales. It is found that the supergiant shell LMC 4 is about $1 \mathrm{kpc}$ in diameter. The size of the supergiant shell can be considered to be typically the area covered by propagating star formation. Therefore, we have chosen very similar length scale for identifying the star clusters.

\section{Estimation of star formation history}

The star formation history of the regions studied is derived using the CMDs. The CMDs represent the stellar populations of different ages present in the region. The brightest mainsequence (MS) stars represent the stars born out of the recent star formation event and the red giants represent stars born from earlier star formation events. We use the technique of synthetic CMDs to reproduce the observed CMD and thereby estimate the star formation history.

\subsection{Observed CMDs}

The observed $V$ vs. $(B-V)$ CMDs are obtained for stars located within a radius of a few arcmin from the center. The CMDs are shown in Fig. 2. The CMDs are affected by the reddening towards the observed region, photometric incompleteness in the data which is a function of stellar crowding and stellar magnitudes, and the distance. The incompleteness in the data is 
Table 2. Statistics of star clusters identified near the regions.

\begin{tabular}{lccrrrrr}
\hline \hline Region & $\begin{array}{c}\text { No. of clusters } \\
\text { within 30 arcmin }\end{array}$ & $\begin{array}{c}\text { No. of clusters } \\
\text { with age known }\end{array}$ & $\leq 7.5$ & $7.5-8.0$ & $8.0-8.5$ & $8.5-9.0$ & $\geq 9.0$ \\
\hline Region 1 & 15 & 12 & 1 & - & 7 & 3 & 1 \\
Region 2 & 9 & 8 & - & 4 & 1 & 2 & 1 \\
Region 3 & 17 & 13 & - & 3 & 3 & 3 & 4 \\
Region 4 & 24 & 19 & - & 2 & 11 & 4 & 2 \\
Region 5 & 17 & 15 & 1 & 1 & 6 & 5 & 2 \\
Region 6 & 27 & 22 & - & 4 & 11 & 7 & - \\
\hline
\end{tabular}
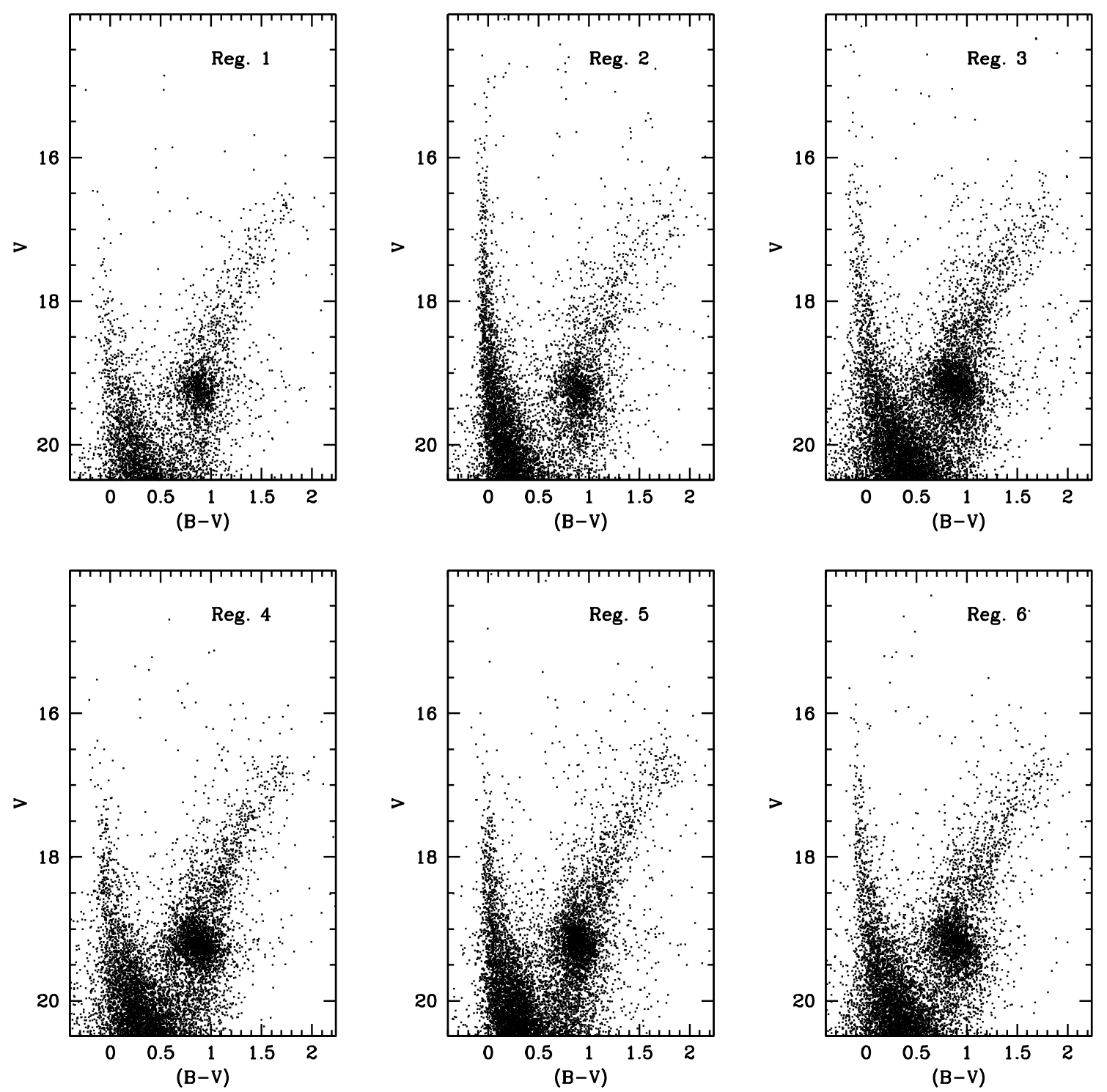

Fig. 2. The $V$ vs. $(B-V)$ CMDs of all the six regions studied here are shown. These CMDs are used to estimate the stellar density after correcting for data incompleteness.

tabulated in Udalski et al. (2000) and these values are used for correcting the incompleteness. The CMD is made into a two dimensional array by binning in $V$ and $(B-V)$. The width of the bin in $V$ is $0.2 \mathrm{mag}$ and in $(B-V)$ it is $0.1 \mathrm{mag}$. The number of stars in each bin is counted and then corrected for the incompleteness. For each box in the CMD there would be separate values for the data completeness corresponding to the $V$ and $B$ frames, and the smaller value was used for the correction. After correcting for the incompleteness, the two-dimensional stellar count was obtained. The number density of stars in each 
box is estimated by normalising the above values with respect to the total number of stars in the CMD. This density distribution of stars in the CMDs is used to compare with the synthetic CMDs. The selection of the values for reddening and the distance modulus are discussed in Sect. 3.3.

\subsection{Synthetic CMDs}

The synthetic CMDs are made with the help of stellar evolutionary models after assuming a model for the age-metallicity relation. We used the evolutionary models of the Padova group, Fagotto et al. (1994a,b). The following relation between age and metallicity was found to be satisfactory: for ages less than 4 Gyr, we used the $Z=0.008$ models; for ages between 4 and 9 Gyr we used $Z=0.004$ models and for ages more than 9 Gyr and up to $12 \mathrm{Gyr}$; we used $Z=0.0004$ models. A small fraction of binaries $(\sim 10 \%)$ and the photometric error in the observation are also included in the synthetic CMDs. The algorithm used here is an extension of that used in Subramaniam \& Sagar (1995, 1999). In this algorithm, we introduced the age range such that we obtain a heterogeneous population in age.

The procedure is described below. Synthetic CMDs were created for a set of ages with small age ranges. The stellar distribution in the synthetic CMDs was converted to a density distribution, using a procedure similar to the observed CMD. These synthetic CMDs were used as templates of the stellar density distribution for various age ranges. The synthetic CMDs were created with a sufficiently high number of stars to minimise the statistical fluctuations. The above mentioned templates were created for 10-50 Myr, 51-100 Myr and then 9 templates between 100 and $1000 \mathrm{Myr}$, in steps of $100 \mathrm{Myr}$, thereby creating 11 templates. For the $50 \mathrm{Myr}$ age range, the stars were created with steps of $5 \mathrm{Myr}$, whereas for the $100 \mathrm{Myr}$ age range, a step of $10 \mathrm{Myr}$ was used. For ages beyond $1 \mathrm{Gyr}$, the templates were created for an age range of $200 \mathrm{Myr}$, up to $2 \mathrm{Gyr}$, such that 5 templates were made available. Between 2 and 5 Gyr, 6 templates were created with an age range of 500 Myr. Though the present data is very suitable for estimating the star formation history beyond $5 \mathrm{Gyr}$, for the sake of completion, the templates for ages older than $5 \mathrm{Gyr}$ were also included. For older ages, the templates were made for 5-6 Gyr, 6-8 Gyr, 8-10 Gyr and 10-12 Gyr. These templates were used to create the final CMD in terms of stellar density, which in turn was compared with the density of the observed CMD. The densities of the templates are scaled and combined to obtain the best fitting synthetic CMD, based on the $\chi^{2}$ minimisation technique. Scaling the stellar density in various templates is equivalent to adjusting the star formation rates (SFRs) at the respective ages. The SFRs are estimated in units of $1 \times 10^{-5} M_{\odot} \mathrm{yr}^{-1}$, such that this is the minimum detectable value. The range in the scaling factor for which the minimum value of the $\chi^{2}$ obtained is estimated for each template. The average and the deviation about the mean of the star formation rate for each age range are thus estimated.

The technique used here is very similar to that used in Dolphin (1997), Olsen (1999) and Dolphin (2000). For stars younger than 1 Gyr the resolution in age is higher, whereas it is lower for populations older than 1 Gyr. As we attempt to compare the cluster formation episodes, which have higher resolution at younger ages, the above resolution values are adequate. The limiting magnitude in the OGLE II data is around $V=21.0 \mathrm{mag}$. This implies that the stars on the MS are younger than about 1.6 Gyr, while the RGB stars are a mixture of both young and old population. Therefore the present data is not suitable for understanding the star formation history for stars older than 4 Gyr. Also the clusters are known to show an age gap between 4 and 10 Gyr. Although we have considered templates up to $12 \mathrm{Gyr}$, the comparison between the star and the cluster formation episodes stops at $4 \mathrm{Gyr}$.

\subsection{Reddening and distance modulus}

The reddening towards each region is estimated by comparing the location of the observed MS stars with the MS of the templates younger than $1 \mathrm{Gyr}$. The comparison was made with the stellar CMD. Regions 1,3 , and 4 were found to have a reddening of $E(B-V)=0.06 \mathrm{mag}$, regions 2 and 5 were found to have $E(B-V)=0.12 \mathrm{mag}$ and region 6 was found to have $E(B-V)=0.08 \mathrm{mag}$. The reddening estimates have an error of $0.02 \mathrm{mag}$. These reddening values agree very well with the reddening estimates found by Subramaniam (2003) for various locations in the bar region, which are similar to the estimates by Udalski et al. (1999), but with better spatial resolution. These values are also consistent with the values previously estimated for other locations in the LMC and agrees (within the errors) with those estimated by Dolphin (2000). Following Pietrzynski \& Udalski (2000) a distance modulus of $18.24 \mathrm{mag}$ for the LMC is assumed initially. While creating the synthetic CMDs, the value of the distance modulus was changed and the value giving rise to the minimum value of $\chi^{2}$ is adopted. A value of $18.30 \pm 0.06$ was found to produce the best fit for all the six regions. This would indicate a distance of $45.7 \pm 1.3 \mathrm{kpc}$ to the LMC. For this value of the distance modulus, 1 arcmin corresponds to $13.4 \mathrm{pc}$ in the LMC. Thus 30 arcmin would correspond to $400 \mathrm{pc}$. Thus the region scanned for clusters is $800 \mathrm{pc}$ in diameter, this is similar to the sizes of supergiant shells in the LMC.

\section{Results}

\subsection{Region 1}

15 star clusters were identified within a radius of $30 \mathrm{arcmin}$ $(\sim 400 \mathrm{pc})$, for 12 of these the ages are known. We find that $58 \%$ of the clusters in this region have ages between 100-300 Myr, and $25 \%$ between $300 \mathrm{Myr}$ and $1 \mathrm{Gyr}$. Only $8 \%$ of the clusters are either younger than $30 \mathrm{Myr}$ or older than $1 \mathrm{Gyr}$. Hence the bulk of cluster formation has occurred from 100-300 Myr ago with a tapering rate towards 1 Gyr. Figure 5 shows the histogram of the normalised fraction of clusters with respect to age. The CMD of this field consists of 4132 stars within a radius of 5 arcmin as shown in Fig. 2. The observed stellar density is shown in Fig. 3, along with the synthetic one. It can be seen that the synthetic CMD looks very much like the observed 

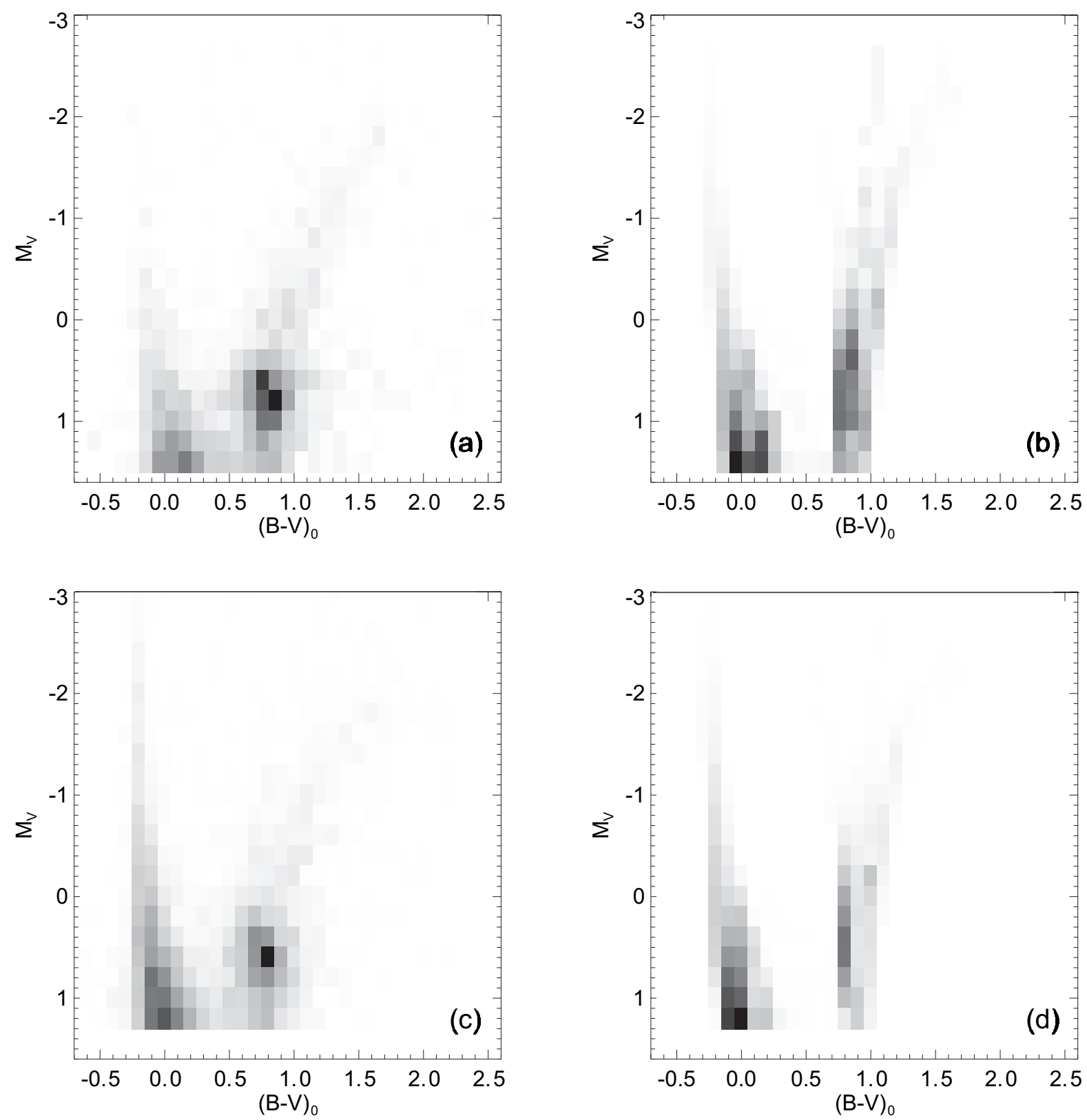

Fig. 3. The stellar densities on the CMDs for regions 1 and 2. Panels a) and c) correspond to the observed CMDs, b) and d) correspond to the synthetic CMDs for regions 1 and 2 respectively.

one. The part which is not reproduced is the very high density of the red clump stars. The other minor effect is the marginally higher number of stars seen at the faint end of the MS. The overall agreement is quite good and the reduced $\chi^{2}$ value of the fit is 0.07 . The estimated star formation rates are shown in Fig. 4. The region is found to have had a higher rate of star formation between 500-1000 Myr ago, then a reduced star formation until 2 Gyr ago. Between 2 Gyr to 10 Gyr, there was star formation at a very low rate. This region shows the presence of a burst of star formation between 10-12 Gyr. Also, we see that this region did not form many stars in the last $500 \mathrm{Myr}$.

Figure 5 shows the comparison of SFRs and fraction of clusters formed in various age groups. It is seen that the star formation event between 1.0 and $2.0 \mathrm{Gyr}$ resulted in the formation of one star cluster. The star formation which continued to younger ages, resulted in the formation of 3 star clusters, which fall in the age range $300 \mathrm{Myr}-1 \mathrm{Gyr}$. The enhancement in the cluster formation between $100 \mathrm{Myr}-300 \mathrm{yr}$ is not reflected in the formation of field stars. For ages older than $300 \mathrm{Myr}$, the cluster and star formation events are correlated such that the number of clusters found in the age group 1-2 Gyr is lower than that found in the group $300 \mathrm{Myr}-1$ Gyr. On the other hand an anti-correlation is seen for ages younger than $300 \mathrm{Myr}$. Also there is very low star formation in the last $100 \mathrm{Myr}$, whereas there are at least two star clusters younger than $100 \mathrm{Myr}$. The point to be noted here is that the cluster formation seems to have continued to very recent times, whereas low SFRs are found in the last 200 Myr. In particular, the star formation and the cluster formation rates seem to differ in the last 100-300 Myr history. 

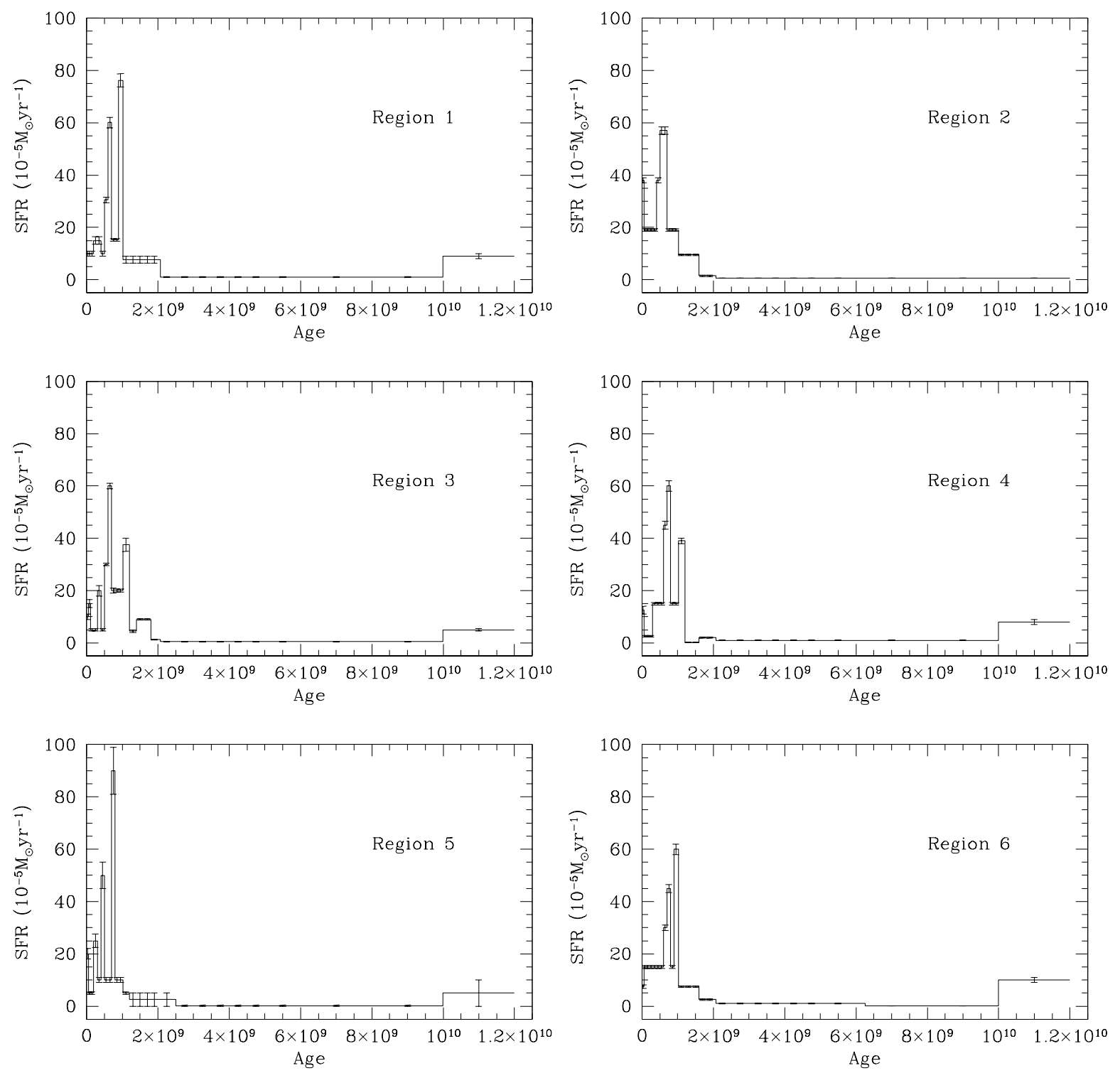

Fig. 4. The estimates of SFRs in the six regions between $10 \mathrm{Myr}$ to $12 \mathrm{Gyr}$ are plotted as histograms.

\subsection{Region 2}

In this region, 9 star clusters have been identified within 30 arcmin radius and the ages of 8 clusters are known. $50 \%$ of the clusters have ages in the range 30-100 Myr, $12.5 \%$ have ages in the range of 100-300 Myr, 25\% in the range $300 \mathrm{Myr}-1 \mathrm{Gyr}$ and $12.5 \%$ have ages beyond $1 \mathrm{Gyr}$. The field star population within 6 arcmin $(80 \mathrm{pc})$ radius is studied based on a CMD of 6919 stars. The CMD is shown in Fig. 2.

The estimated SFRs show that this region has experienced a substantial increase in the SFR at ages around $500 \mathrm{Myr}$, after that the rate has decreased to near zero values at about 2 Gyr. Between 2-12 Gyr, there was very little star formation in this region. For younger ages, the star formation has continued till about 10 Myr. There has been a small increase in SFR in the last 50 Myr. Therefore this region has had continuous star formation in the last 2 Gyr period. The observed stellar density is shown in Fig. 3, along with the synthetic one. The feature which is not reproduced in the synthetic CMD is the peak in the red clump, as in region 1 . The reduced $\chi^{2}$ value for the fit is 0.06. A small enhancement in the star cluster formation which occurred between $300 \mathrm{Myr}-1 \mathrm{Gyr}$, is well correlated with the high star formation rate at about $500 \mathrm{Myr}$. This region does not show the 100-300 Myr enhancement in the cluster formation, but shows a low formation rate of clusters, which also correlates with the low SFR seen during this period. There is one cluster which is less than $30 \mathrm{Myr}$ old, and we do find an increase in the SFR between 10-50 Myr. Thus the star formation and the cluster formation are more or less correlated in this region.

\subsection{Region 3}

This region is situated right at the center of the LMC Bar. 17 clusters are found in the 30 arcmin radius, of which ages are known for 13 clusters. Cluster formation in this region appears to be at a more or less constant rate until $30 \mathrm{Myr}$. 31\% of 

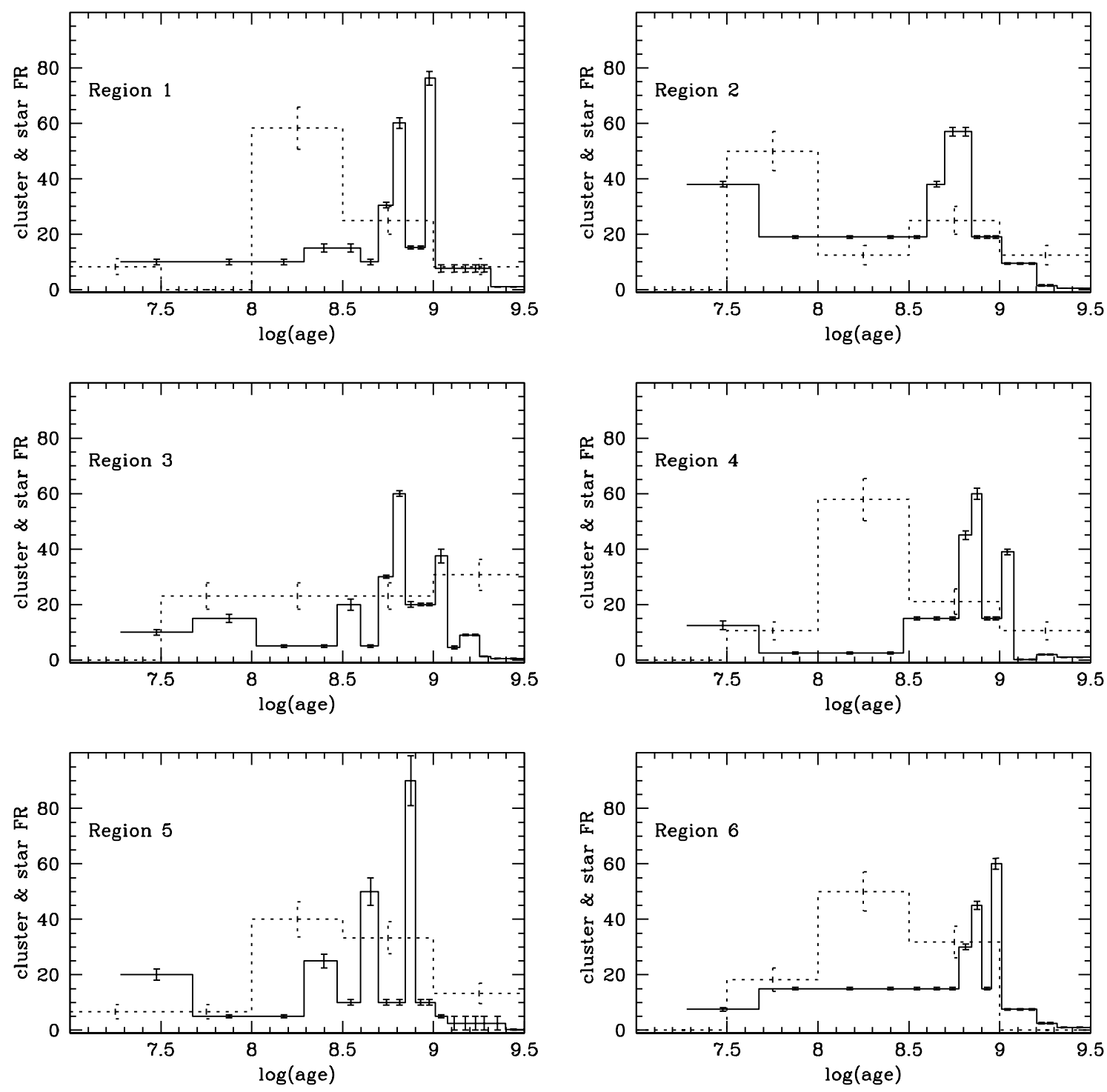

Fig. 5. The SFRs and the fraction of clusters in various age groups are plotted here for six regions. This plot helps to find out correlation between the increases or decreases in the rates of star and cluster formation.

clusters have ages more than $1 \mathrm{Gyr}$, and $23 \%$ of clusters in the lower age ranges. Hence the cluster formation rate was decreased around $1 \mathrm{Gyr}$, after an initial higher rate. The CMD of 9607 stars within a radius of $3 \mathrm{arcmin}$ is shown in Fig. 2.

The SFR estimates show that this region experienced increased SF between 600-1200 Myr. The SFR then decreased and settled at a very low value around 2 Gyr. During the 10-12 Gyr period, this region seems to have experienced an enhancement in star formation. This is similar to region 1, but the estimated SFR is slightly less. The density in the CMDs show that the simulated CMD matches with the observed one well. The red clump peak is again not well reproduced. The reduced $\chi^{2}$ value of the fit is 0.05 . The observed stellar density is shown in Fig. 6, along with the synthetic one.

In this region, we see that the cluster formation was more or less constant, and a steady rate of cluster formation is observed between 30-1000 Myr and a slightly increased rate after 1000 Myr. The estimated SFRs show that the star formation was not continuous between the above period, rather the
SFR increased at $600 \mathrm{Myr}$, then increased further till $1200 \mathrm{Myr}$. Then it is seen to have decreased after $2 \mathrm{Gyr}$. If we consider that the increased star formation seen between 1-1.2 Gyr correlates with the increase in the cluster formation, then both rates can be considered to be correlated for ages older than $1 \mathrm{Gyr}$. Between the ages 300-1000 Myr, the SFR shows an increase and the cluster formation shows a small decrease. On the other hand, for ages in the range 100-300 Myr, we do not find any correlation. The cluster formation is found to have continued at the same rate, whereas the SFR is found to be reduced. The SFR shows an enhancement in the 50-100 Myr age range, with a decreased SFR up to the last 10 Myr. Thus the star and cluster formation rates are found to be more or less correlated, except for the 100-300 Myr age range.

\subsection{Region 4}

There are 24 star clusters within an area of 30 arcmin radius and ages are known for 19 of them. The majority of star 

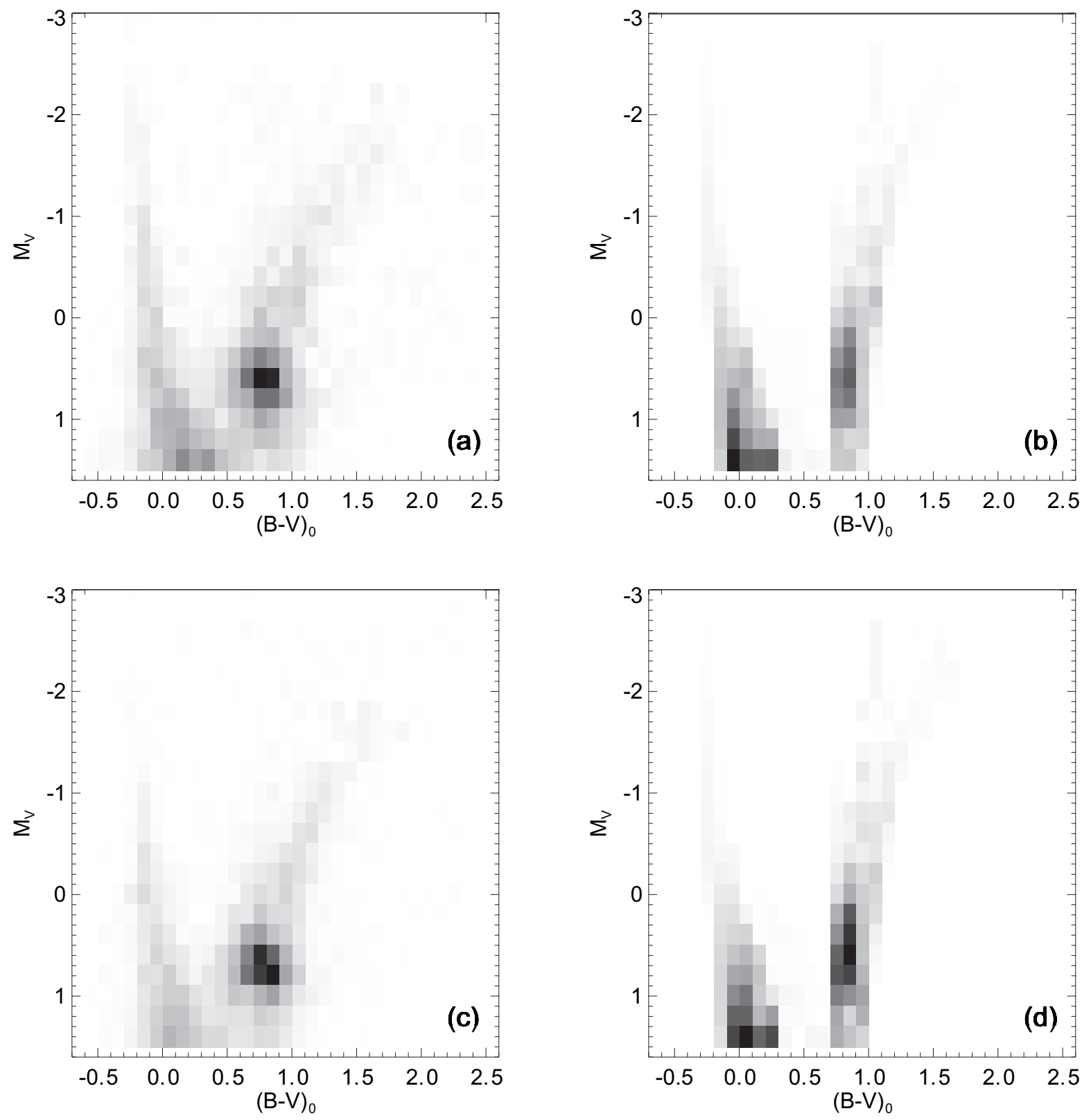

Fig. 6. The stellar densities on the CMDs for regions 3 and 4. Panels a) and c) correspond to the observed CMDs, b) and d) correspond to the synthetic CMDs for regions 3 and 4 respectively.

clusters were formed during the period 100-300 Myr, viz. $58 \% .21 \%$ were formed during the period of $300 \mathrm{Myr}-1 \mathrm{Gyr}$, $10 \%$ during the period of 30-100 Myr and the rest during the period before 1 Gyr. The CMD of stars within 2 arcmin radius plotted in Fig. 2 comprises 5244 stars.

The synthetic CMD as shown in Fig. 6 has more MS stars and a wider RGB. The reduced $\chi^{2}$ value for the fit is found to be 0.1 , which is slightly higher than for the other regions. The SFR shows peak values between $500 \mathrm{Myr}$ and $1.3 \mathrm{Gyr}$. A small enhancement is seen around 2 Gyr. The SFR is found to be very low in the age range $2-10 \mathrm{Gyr}$, with a relatively high rate between 10-12 Gyr. On comparing the star and cluster formation episodes we find that the star formation event which occurred in the age range 1.0-2.0 Gyr has managed to form two star clusters. The star formation which continued to younger ages, up to $300 \mathrm{Myr}$, has resulted in the formation of 4 star clusters. The cluster formation episode is seen to be quite strong in the age range 100-300 Myr, whereas a very low SFR is estimated in the same age range. Two star clusters are found to be younger than $100 \mathrm{Myr}$, and an enhancement in the SFR is found for ages younger than $50 \mathrm{Myr}$. Thus the star and cluster formation rates are well correlated except for the age range 100-300 Myr.

\subsection{Region 5}

Within a radius of $400 \mathrm{pc}$, we identified 17 star clusters and ages are known for 15 of them. We find that $40 \%$ of the clusters have ages in the range 100-300 Myr, 33.3\% have ages in the range $300 \mathrm{Myr}-1 \mathrm{Gyr}$ and $13.3 \%$ are older than $1 \mathrm{Gyr}$. 

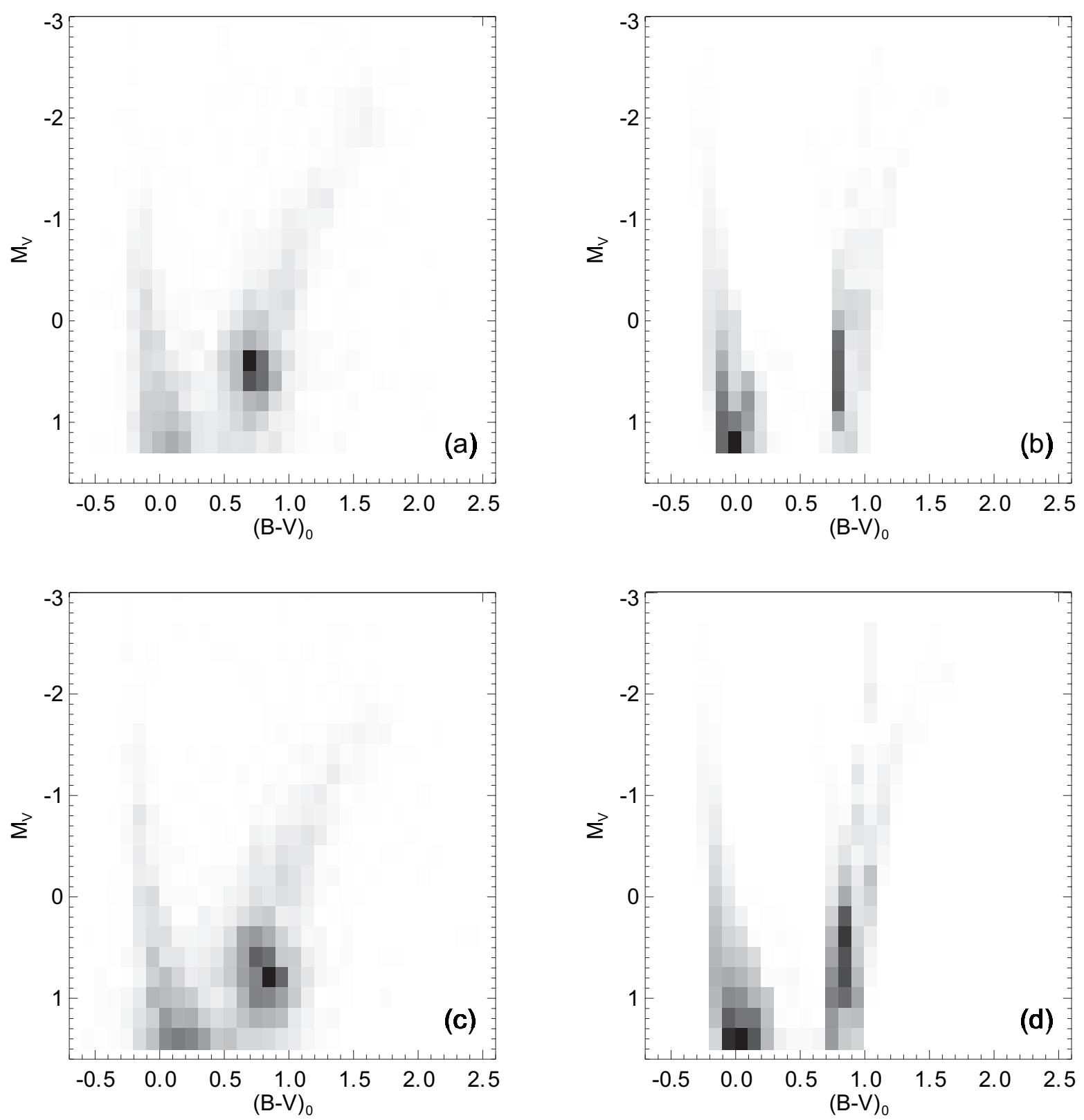

Fig. 7. The stellar densities on the CMDs for regions 5 and 6. Panels a) and c) correspond to the observed CMDs, b) and d) correspond to the synthetic CMDs for the regions 5 and 6 respectively.

The CMD of 6503 field stars within a region of $2 \operatorname{arcmin}(27 \mathrm{pc})$ radius is shown in Fig. 2.

The synthetic and observed CMDs are compared in Fig. 7. The synthetic CMD shows a vertical RGC and more stars in the fainter end of the MS, which are not seen in the observed CMD. The reduced $\chi^{2}$ value for the fit is found to be 0.14 and this is the worst fit among all the regions. The estimated SFRs show that the values are high in the age range 300-800 Myr. A very small SFR is found between $2-8$ Gyr and a medium rate between 8 and 12 Gyr. A comparison of the cluster formation rate shows that, the two clusters older than 1 Gyr could have been formed between 1-2 Gyr along with the star formation. The enhanced cluster formation between $300 \mathrm{Myr}-1 \mathrm{Gyr}$ is well correlated with the enhancement in the SFR. Six star clusters are formed between 100-300 Myr, whereas an enhancement in the
SFR is found at $\sim 250 \mathrm{Myr}$. One cluster is found to be younger than $30 \mathrm{Myr}$ and one between 30-100 Myr. Correspondingly, enhancement in the SFR is found for ages younger than $50 \mathrm{Myr}$. Hence we find a more or less correlated star and cluster formation for all ages.

\subsection{Region 6}

27 star clusters are found within an area of 30 arcmin radius, of which the ages are known for $22.18 \%$ of the clusters have ages within 30-100 Myr, 50\% have ages between 100-300 Myr and 32\% have ages between $300 \mathrm{Myr}-1 \mathrm{Gyr}$. These indicate that there was a burst of cluster formation in the period 100-300 Myr ago, with a tapering rate before and after this burst. 3302 field stars located within $4 \operatorname{arcmin}(54 \mathrm{pc})$ 
radius were identified and the CMD of the field stars is plotted in Fig. 2.

The synthetic CMD in Fig. 7 shows that the RGC population is not matched, though the overall match could be considered satisfactory. The reduced $\chi^{2}$ value is found to be 0.06 . The estimated SFRs are found to be high in the interval of $50 \mathrm{Myr}-1000 \mathrm{Myr}$, and then decreasing up to 2 Gyr. A very low SFR is found in the interval of 2-10 Gyr, with a relatively higher rate in the 10-12 Gyr interval. A comparison of the SF and the CF episodes shows that both the rates are found to increase at about $1 \mathrm{Gyr}$. The $\mathrm{CF}$ rate increases further at $300 \mathrm{Myr}$, whereas the SF rate is reduced. This region is found to show recent star formation corresponding to the presence of young star clusters. 4 star clusters are found in the age range 30-100 Myr, whereas the SFR is found to decrease slightly at $50 \mathrm{Myr}$. Therefore, the SF and CF are found to be less correlated in the age range 30-100 Myr, whereas indications of anti-correlation are found in the 100-300 Myr age range.

\section{Discussion}

The aim of the present analysis is to verify the correlation between the star and cluster formation rates at different epochs in the LMC, particularly on smaller scales. The present study shows that SFRs and the cluster formation rates are more or less correlated for the age range 30-100 Myr. In the age range 100-300 Myr, 5 regions show an enhancement in cluster formation, whereas such an enhancement is not noticed in the SFR. In the age range of 300-1000 Myr, the SF as well as the cluster formation shows enhanced in rates. It is also found that the SF and CF are more or less correlated for ages more than 1 Gyr.

Girardi et al. (1995) estimated the age distribution of star clusters in the LMC. They found three periods of enhancement in the formation of star clusters in the LMC, namely at $0.1 \mathrm{Gyr}$, 1-2 Gyr and 12-15 Gyr. Pietrzynski \& Udalski (2000) found peaks in star cluster formation at $7 \mathrm{Myr}, 125 \mathrm{Myr}$ and $800 \mathrm{Myr}$. They also found peaks at $100 \mathrm{Myr}$ and $160 \mathrm{Myr}$, which they attribute to the last encounter between the Magellanic Clouds. Grebel et al. (1999) found that the age distributions of both the LMC and the SMC clusters peak at $100 \mathrm{Myr}$, when the Clouds had their closest encounter and last perigalacticon. All the above results indicate that the substantial number of clusters seen in the 100-300 Myr around 5 regions could be part of the cluster formation peak at that time. Hence the anti-correlation between the SF and CF during this period could indicate that the type of SF which induced the CF is different in this period. On the other hand, the peak in the CF at $800 \mathrm{Myr}$ is well correlated with the SF, as in most of the regions, the SFR was highest around the age of $800 \mathrm{Myr}$. Therefore, the highest correlation between $\mathrm{CF}$ and SF rates is found at $\sim 800 \mathrm{Myr}$, whereas anticorrelation is found in the age range of 100-300 Myr.

It is possible that some assumptions as well as the choice of data could modulate or bias the results obtained here. Some of the possibilities are explored below. (1) Incompleteness in the cluster data: the incompleteness affects the fainter clusters and hence the older clusters will be more affected than the younger ones with bright stars. This can only increase the discrepancy observed here and not reduce it. (2) Inappropriate estimates of the SFRs: an inspection of the CMDs of the regions presented in Fig. 2 indicates low star formation rates for ages younger than a few hundred Myr. In most of the cases the brightest few stars observed belong to the $100 \mathrm{Myr}$ age range. There could be bias for very bright stars as they could have been saturated in the images. This can only affect the very young population, but not for ages older than 50 Myr. (3) Statistical significance: the result obtained here is statistically significant as it is found for 5 of the 6 regions studied. All the regions have a good number of star clusters especially for ages younger than $300 \mathrm{Myr}$. (4) Selection effects: there was no a priori information for the regions studied here and they were not selected because of a particular property. The choice was only based on the availability of clusters in the neighbourhood. Hence the results are very unlikely to be affected by this. (5) The center field may not be representative for the entire $400 \mathrm{pc}$ region. We have assumed that the stars in the central few pc could represent the whole area with a radius of $400 \mathrm{pc}$. If this assumption is not valid such that there is difference in population within the radius, the results above may not be valid. To check this, the CMDs were created for stars located in the annulus with 25 and 30 arcmin as the inner and outer radii, which sampled the stars near the periphery. When the central and the peripheral CMDs were compared, no significant changes in the stellar density were noticed. Special attention was given to the younger population and no noticeable increase was noticed in the stellar population younger than $300 \mathrm{Myr}$. Hence we assert that the result obtained here is likely to be a true feature in the LMC, at least near the bar regions.

In general star formation results in the formation of field stars as well as groups or clusters of stars. Therefore, the SFR is an indicator of the amount of star formation or the major star formation events. The rate of cluster formation therefore is expected to be correlated with the SFR. The main result of this study is that the SF and CF are not seen to be correlated in the 100-300 Myr history of the LMC, whereas it seems to be well correlated for ages higher as well as lower than the above range. This is a surprising result as one expects the imprint of star formation to be present in the field stars as well.

The results presented here could indicate that the star formation in the LMC has a preference to the formation of clusters, bound or unbound in the 100-300 Myr age range. This results in an overabundance of star clusters in this age range. Such a discrepancy decreases for older ages, as stellar evolution as well as dynamical evolution dissolves the clusters beyond the limit of detection. This happens for open clusters and not for the blue globulars. The LMC is also seen to have a healthy population of binary clusters. The age distribution of the multiple clusters in the LMC shows that the largest fraction falls in the 100-300 Myr age range. This also supports the idea of preferred formation of clusters. As the LMC is known to have a slowly rotating disk, the clusters born together are likely to spend a considerable amount of time together. As the tidal field of the LMC is very weak, the clusters located close to each other have a better chance to merge than to disrupt.

The LMC is known to be interacting with our Galaxy and the Small Magellanic Cloud (SMC). There are many studies 
that have looked for the signatures of possible encounters (Westerlund 1997, and references therein; Maragoudaki 2001) and also studies which have done simulations of the dynamics of the interaction between the three galaxies (Fujimoto \& Murai 1984; Gardiner \& Noguchi 1996; Gardiner et al. 1994). These studies have found that the LMC interacted with our Galaxy at about $1.5 \mathrm{Gyr}$ ago, with the SMC at $0.2-0.4 \mathrm{Gyr}$ and that the LMC had a perigalacticon passage at $100 \mathrm{Myr}$. Thus the cluster formation episode of 100-300 Myr could be correlated with the interaction with the SMC or our Galaxy. The SMC also shows a peak in cluster formation between 100-300 Myr (Grebel et al. 1999). Some clusters could also have been formed due to the propagating star formation started by the triggers. On the contrary, the interaction between the Clouds does not seem to be the cause for the cluster and star formation peak at $\leq 1000 \mathrm{Myr}$. The result presented here indicates that the star formation induced by interaction may likely to be biased towards group formation of stars, whereas such a bias is not found in the case of star formation without any interaction. Formation of globular clusters or dense clusters is also observed in interacting galaxies. For example, in the colliding galaxies, NGC 4038/4039 very massive clusters are found to be formed.

Acknowledgements. I thank the referee G. Pietrzynski for helpful comments.

\section{References}

Bica, E., Claria, J. J., Dottori, H., Santos, J. F. C. JR., \& Piatti, A. E. 1996, ApJ, 102, 57

Bica, E., Schmitt, H. R., Dutra, C. M., \& Oliveira, H. L. 1999, AJ, 117,238

de Boer, K. S., Braun, J. M., Vallenari, A., \& Mebold, U. 1998, A\&A, 329, L49
Dolphin, A. E. 2000, MNRAS, 313, 281

Dolphin, A. 1997, New Astron., 2, 397

Fagotto, F., Bressan, A., Bertelli, G., \& Chiosi, C. 1994a, A\&A, 105, 29

Fagotto, F., Bressan, A., Bertelli, G., \& Chiosi, C. 1994b, A\&A, 104, 365

Feitzinger, J. V., Glassgold, A. E., Gerola, H., \& Seiden, P. E. 1981, A\&A, 98, 371

Fujimoto, M., \& Murai, T. 1984, IAU Symp., 108, 115

Gardiner, L. T., \& Noguchi, M. 1996, MNRAS, 278, 191

Gardiner, L. T., Sawa, T., \& Fujimoto, M. 1994, MNRAS, 266, 567

Girardi, L., Chiosi, C., Bertelli, G., \& Bressan, A. 1995, A\&A, 298, 87

Grebel, E. K., Zaritsky, D., \& Harris, J. 1999, IAU Symp., 190, 405

Holtzman, J. A., Gallagher III, J. S., Cole, A. A., et al. 1999, AJ, 118, 2262

Maragoudaki, F., Kontizas, M., Morgan, D. H., et al. 2001, A\&A, 379, 864

Olsen, K. A. G., Hodge, P. W., Mateo, M., et al. 1998, MNRAS, 300, 665

Olsen, K. A. G. 1999, AJ, 117, 2244

Pietrzynski, G., Udalski, A., Kubiak, M., et al. 1999, Acta Astron., 49, 521

Pietrzynski, G., \& Udalski, A. 2000, Acta Astron., 50, 337

Searle, L., Wilkinson, A., \& Bagnoulo, W. 1980, ApJ, 239, 803

Subramaniam, A. 2003, ApJ, 598, L19

Subramaniam, A., \& Anupama, G. C. 2002, A\&A, 390, 449

Subramaniam, A., \& Sagar, R. 1995, A\&A, 297, 695

Subramaniam, A., \& Sagar, R. 1999, AJ, 117, 937

Udalski, A., Soszynski, I., Szymanski, M., et al. 1999, Acta Astron., 49, 223

Udalski, A., Szymanski, M., Kubiak, M., et al. 2000, Acta Astron., 50, 307 (OGLE II data)

van den Bergh, S. 1999, IAU Symp., 190, 569

Westerlund, B. E. 1997, The Magellanic Clouds (Cambridge: Cambridge Univ. Press)

Zaritsky, D., Harris, J., \& Thompson, I. 1997, AJ, 114, 1002 